\title{
ESTADO DE LA BIODIVERSIDAD TERRESTRE DE LA REGIÓN AUTÓNOMA ATLÁNTICO SUR (RAAS), NICARAGUA
}

Christopher A. Jordan ${ }^{[1]}$ MSc. Kirkman Roe Hulse ${ }^{[2]}$

\section{Resumen}

La Región Autónoma Atlántico Sur (RAAS) de Nicaragua aparece con frecuencia en la literatura académica a raíz de su alta diversidad cultural y biológica, y por su reconocimiento como uno de los últimos lugares remotos del mundo. No obstante, la biodiversidad terrestre de la región carece de representación en las publicaciones científicas, las cuales se limitan a perspectivas generales no basadas en datos científicos, estudios de la etnobotánica e investigaciones sobre la deforestación o regeneración del bosque. Este artículo presenta un informe de los resultados preliminares de una investigación sobre la diversidad de los mamíferos y aves terrestres, basada en el uso de cámaras con sensores de movimiento. Asimismo, se refiere tanto la literatura histórica como la literatura contemporánea de la zona para explorar las implicaciones potenciales. La evidencia sugiere que la recopilación de datos básicos sobre la biodiversidad regional debe ser una prioridad en la etapa actual, durante la cual varias fuerzas locales y globales van conectando y cambiando la Costa Caribe; convirtiéndola en un lugar no tan remoto, y sí sostenible y amigable a la fauna silvestre como aparece en la mayoría de la literatura.

\section{Introducción}

En los últimos 50 años, un flujo básicamente continuo de investigadores llegan a la Región Autónoma Atlántico Sur (RAAS) de Nicaragua con el fin de estudiar su diversidad biocultural. La región tiene ecosistemas altamente diversos, incluyendo la selva baja, los manglares, los bosques inundados, y ecosistemas de importancia ecológica y económica como son las lagunas, los estuarios, y las áreas marinas (Sollis, 1989). También hay una impresionante diversidad de pueblos. La Costa alberga seis etnicidades distintas: los krioles, los garífuna, los miskito, los rama, los ulwa, y los mestizos. Es muy común que los habitantes puedan hablar entre tres y cinco idiomas.

[1] Michigan State University. Department of Fisheries and Wildlife. 13 Natural Resources-East Lansing, Michigan, 48824 USA. Corresponding Author, E- mail: jordan41@msu.edu

[2] Master en ciencias, docente Universidad de las Regiones Autónomas de la Costa Caribe Nicaragüense (URACCAN) recinto Bluefields, Correo: kirkman.joeroe1o@gmail.com 
En un sentido general, los investigadores en distintas disciplinas académicas han dado a la Costa Caribe de Nicaragua la reputación internacional como uno de los últimos lugares remotos del mundo donde: "las condiciones demográficas e infraestructurales...hace que la recopilación de datos de censo fidedignos de cualquier tipo, sea extraordinariamente difícil" (Jamieson, 1999). La Costa Caribe estaba aislada de los mercados nacionales principales de la Costa Pacífica durante la mayoría de su historia por la cordillera que biseca a Nicaragua y por la falta de infraestructura adecuada. La región carece de un sistema moderno de carreteras, por lo cual los medios de transporte acuático son los más comunes. Es dentro de este contexto que muchos comunitarios se dedican todavía a la extracción de recursos naturales, tanto terrestres como marinos, y la agricultura de subsistencia. En particular, hay una cultura marinera muy fuerte a raíz de la dependencia en la cosecha, venta local e intercambio de chacalines, manatíes, tortugas y pescado o trueques. La pesquería de la zona afecta a varias especies amenazadas y en vías de extinción según la Lista Roja de la Unión Internacional para la Conservación de la Naturaleza y los Recursos Naturales (IUCN), por lo cual la pesquería aparece con frecuencia en la literatura académica (Archbold, 2008; Campbell, 2003, Garland \& Carthy 2010; Jamieson, 2002; Jimenez, 2002; Nietschman, 1973). Informes sobre la sostenibilidad de la agricultura del "roza, tumba y quema" que practican muchos costeños también son relativamente comunes (Jamieson, 2010; Nietschmann, 1972; Roe, Working, Paper).

No obstante, han sido varias etapas durante las cuales la RAAS ha albergado empresas internacionales con el deseo de extraer y acrecentar ganancias de la madera preciosa, tortugas marinas, y pescados (Nietschmann, 1973). Estas empresas contrataban a los comunitarios de la RAAS y les daban sueldos fijos y productos del mercado, pero siempre de un modo temporal. No se estableció vínculos permanentes con los mercados y sistemas globales. Además, aunque algunos costeños se convirtieron en capitalistas, en un sentido general, el aprovechamiento local de recursos naturales no seguía una tendencia capitalista y la sobre explotación a escala de los mercados internacionales se ha limitado a los períodos con presencia de empresas extranjeras.

Sin embargo, en el 2007 con la construcción de una carretera, la comunidad de Laguna de Perlas se conectó a Managua a través de El Rama, una ciudad con puerto fluvial. La perspectiva preponderante después de la construcción era el subsiguiente desarrollo, integración de mercados e introducción de tecnología avanzada que contribuirían a detener el deterioro del legado cultural y biológico de la región. Análisis preliminar y al corto plazo describe un aumento en la venta de pescados locales y la disminución del intercambio tradicional de recursos en las comunidades indígenas tradicionales (Garland \& Carthy, 2010; Schmitt \& Kramer, 2010).

La carretera también ha facilitado una afluencia de inmigrantes, en espera para fomentar el avance de la frontera agrícola que se inició en los años 50 y que aumentó con la colocación estratégica de los mestizo quienes se organizaron en cooperativas 
sandinistas agrícolas, dentro de las fronteras de la región costera en la década de 1990. Los nuevos colonos mestizos se han percibido por los pueblos costeros como invasores y en cuanto a las prácticas de uso de la tierra, a diferencia de estos, loss nuevos colonos se dedican a la tala generalizada y la quema de pastizales para el ganado. Esta zona de la RAAS está cambiando rápidamente desde una localidad remota salpicada de pueblos tradicionales de pescadores de subsistencia y horticultores, cambios propiciados por la masiva migración de campesinos, y que a su vez repercute en el sistema ecológico.

Por la diversidad ecológica y los cambios que se generan, la RAAS es un candidato ideal para un plan de monitoreo ecológico a largo plazo. En conjunto a esta importancia, dos razones tuvieron influencia en la decisión del ámbito de este estudio: 1) Los bosques comunales de la región son de alta importancia para la conservación de las poblaciones regionales de la fauna terrestre, como vínculo clave en el Corredor Biológico Mesoamericano. De hecho, constituyen la única conexión ininterrumpida entre el resto del Corazón del Corredor Biológico Mesoamericano en la frontera entre Nicaragua y Honduras al Norte y el Corredor Biológico El Castillo San Juan La Selva que se extiende a ambos lados de la frontera Sur con Costa Rica. Esta conexión podría ser crucial para la migración de especies de gran alcance, tales como jaguares, pumas, tapires (Nombres científicos se encuentran en el Apéndice No. 1) y por lo tanto importante para la conservación de la diversidad genética y resistencia de los ecosistemas regionales. El seguimiento de su funcionalidad como corredor durante todo el proceso actual de desarrollo debe ser una prioridad, y 2) A pesar de la ubicuidad de los investigadores en la RAAS durante los últimos 50 años, por cualquier razón, los informes sobre la diversidad de su fauna terrestre son poco comunes.

La información contemporánea más detallada se encuentra en las publicaciones de los antropólogos (Jamieson, 2010; Loveland, 1967). Nietschmann (1972) es la principal fuente informativa de la información de base, documentó las cazas del jaguar comerciales y listas de especies comúnmente cazadas en Tasbapauni, una pequeña comunidad miskita en las afueras de la gran cuenca de Laguna de Perlas (Figura No. 1). Carr (1956), también describió brevemente la fauna de la región. Los estudios contemporáneos de los bosques y los ecosistemas de pantanos existen, pero se centran generalmente en los árboles, las palmas, o hierbas medicinales, y ofrecen sólo información auxiliar sobre la fauna (Coe 2008, Barret 1998, Vandermeer y Perfecto 2005).

Una excepción a la tendencia general es Cody (2000), quien llevó a cabo un estudio sobre aves seguidora de hormigasen, un área protegida muy al sur. Además Bradford et al (2003) describieron parte de la fauna de la Cuenca del río Escofram. En el contexto del apogeo de la nueva carretera, está pone en evidente peligro las comunidades de mamíferos y la avifauna, asimismo la posibilidad de registrar datos sobre estas comunidades, de ahí la importancia de esta investigación. Para una respuesta parcial a estos problemas, una amplia red de sensores de trampas-cámara, se instaló a partir de principios de mayo de 2009. Los primeros resultados se reportan aquí. 


\section{Materiales y métodos}

\section{Área de estudio:}

Como se mencionó anteriormente el paisaje de la RAAS es una matriz de diversos hábitats de tierras bajas tropicales, impulsadas por una variedad de tipos de suelo y las características geográficas (Christie et al., 2000). Hay paisajes de bosques tropicales latifoliados, bosques pantanosos, bosques de manglares. Es importante destacar que varios huracanes han pasado por la región durante los últimos 30 años y han causado la deforestación. Así, los distintos hábitats de la RAAS están representados en una amplitud de las etapas de sucesión en la mayor parte de la Costa. Históricamente, el clima incluye tanto una estación de lluvias como una estación seca. La mayoría de las aproximadamente 2-4,000 $\mathrm{mm}$ de lluvia cae entre mayo y diciembre (Christie et al., 2000).

Las cámaras se instalaron inicialmente en bosques que utilizan los comunitarios (es decir, no estrictamente protegidos o aislados) a lo largo de aproximadamente 75 $\mathrm{km}$ de la Costa Norte de la RAAS. Las primeras comunidades con cámaras en sus alrededores eran Laguna de Perlas, Kahkabila, Brown Bank, La Fe, San Vicente, Orinoco, Pueblo Nuevo, Kara y Karawala. En un sentido general, los ecosistemas forestales locales son ecológicamente similares en todas estas comunidades. Sin embargo, la información étnica, tamaño de la población, y la distancia de la nueva carretera de Laguna de Perlas son diferentes para cada sitio (Tabla 1).

Tabla No. 1: Información de las comunidades con bosques en donde se encuentran ubicadas las cámaras

\begin{tabular}{llcc}
\hline \multicolumn{1}{c}{ Comunidad } & Etnia & Poblacion estimada & Distancia de LP \\
\hline Pearl Lagoon & Creole & 2540 & $0 \mathrm{~km}$ \\
\hline Kahkabila & Miskito & 497 & $8.5 \mathrm{~km}$ \\
\hline Brown Bank & Creole & 202 & $13.6 \mathrm{~km}$ \\
\hline La Fe & Creole & 110 & $17.85 \mathrm{~km}$ \\
\hline San Vicente & Garifuna & 81 & $23 \mathrm{~km}$ \\
\hline Orinoco & Garifuna & 1,010 & $24 \mathrm{~km}$ \\
\hline Pueblo Nuevo & Mestizo & 300 & $36.5 \mathrm{~km}$ \\
\hline Kara & Ulwa & 200 & $66 \mathrm{~km}$ \\
\hline Karawala & Ulwa & 1,700 & $70 \mathrm{~km}$ \\
\hline
\end{tabular}

Los bosques de todas las comunidades, con la excepción de la comunidad de Kara, debido a su geografía están siendo presionados por una frontera agrícola impulsada por mestizos de originarios de la Costa del Pacífico. Los nuevos inmigrantes se ven 
con mayor facilidad durante los meses de temporada seca, cuando ellos queman los pantanos y los desmontes recientes para cazar iguanas y tortugas de agua dulce y para permitir el crecimiento de pastos, respectivamente. Aunque estos nuevos inmigrantes son percibidos y tratados de manera diferente en Pueblo Nuevo, por razones obvias, incluso algunos habitantes de esta comunidad mestiza han expresado su preocupación por la deforestación excesiva para el pastoreo de ganado. Sin embargo, ese sentimiento es mucho más fuerte en las comunidades tradicionales e indígenas; en ocasiones ha conducido a la violencia política y física.

Después, las cámaras se instalaron en las riberas de pantanos, manglares y los bosques poco frecuentados por miembros de las comunidades. Estos incluyen cámaras a lo largo de los ríos de Patch, Nari, y Wawashang (Figura No. 1) y en el banco de arena de tierra que separa la Laguna de Perlas del Mar Caribe. A partir de este año 2010, más cámaras se instalan en comunidades al Sur de Bluefields y en las regiones más remotas al Sur de Punta Gorda.

\section{Capturas de cámaras}

Una red de cámaras de marca Bushnell se instaló a lo largo de este tramo de $75 \mathrm{~km}$ de la Costa a partir de noviembre de 2009. Los objetivos de los esfuerzos son múltiples: 1) crear un inventario de los mamíferos terrestres y avifauna de la RAAS, y 2) evaluar la reputación de la región como un refugio para la fauna en el contexto de un análisis de los impactos de la carretera, las comunidades indígenas y tradicionales, y la frontera agrícola sobre la vida silvestre para informar los futuros esfuerzos de monitoreo y conservación. Teniendo en cuenta los objetivos, las cámaras se fijan principalmente en los ecosistemas forestales utilizados por las comunidades y se encuentran en un gradiente de distancias (en este informe, $\langle 1>75 \mathrm{~km}$ ) desde la nueva carretera de Laguna de Perlas. Esto se basa en el supuesto de que, dada la falta de caminos formales a lo largo de la costa, en igualdad de otras condiciones, mientras más distancia de la carretera, menos rápido y completo será el desarrollo de una comunidad. Como se describe en la sección anterior, hay evidencia anecdótica que apoya esta hipótesis. Los bosques y comunidades específicos de la comunidad fueron elegidos para coincidir con las comunidades muestreadas en el verano de 2009 y 2010 con una encuesta socioeconómica de hogares, parte de un estudio más amplio, se basa en la misma hipótesis sobre la distancia de la carretera.

La selección del sitio específico de las cámaras adentro de los bosques se realiza mediante un método de muestreo en que se divide el paisaje boscoso que rodea a cada comunidad en su propio mapa cuadriculado de $4 \mathrm{~km}^{2}$ células. Las células en 
cada mapa se le asignan un número al azar. A la llegada del investigador Christopher a. Jordan (CAJ) a una comunidad, guías locales (baqueanos) son contratados para ayudar a navegar hasta el punto centro de la célula con el menor número al azar en el bosque local. La primera cámara se instala adentros de esta célula. La segunda cámara se instala en la célula contigua a la primera célula que tiene el siguiente número más bajo al azar. Esto se repite según sea necesario. El diseño pretende ser al azar, sin embargo, toscamente estratificada por la distancia. Por lo general no más de 3 cámaras se instalan en un día determinado. No obstante, las cámaras de cada comunidad se rotan aproximadamente cada 2 meses, entre 3 juegos de células, para lograr una mayor cobertura espacial de los bosques locales. La intención inicial fue muestrear el mismo número de células en cada bosque comunitario, pero los problemas de accesibilidad prohibió esto y el número de células muestreadas varía de 3-8 entre las comunidades.

Los sitios o lugares precisos de la cámara dentro de cada $4 \mathrm{~km}^{2}$ por célula, son elegidos por el guía local o baqueano. Se les pide que escojan un punto en los alrededores del punto céntrico de la célula en el que se cree que la mayor diversidad y cantidad de animales va a pasar. Se eligió este método para garantizar sitios estructuralmente distintos y sitios tanto dentro y fuera de los caminos del bosque. Esto es importante en los estudios de trampas de cámara con el objetivo de hacer inferencias imparciales acerca de comunidades de animales. Las probabilidades de detección con trampas de cámara varían para distintas especies en distintas maneras en función de la estructura del sitio (Harmsen et al. 2009). Entre 13 a 37 cámaras fueron desplegados continuamente desde noviembre 2009 a agosto 2009 en un total de 75 sitios (Figura No. 1).

El diseño también ordena que una distancia de por lo menos $2 \mathrm{~km}$ se mantenga entre todas las cámaras, para ayudar a asegurar que los sitios son independientes. Aunque es probable que la supuesta independencia completa no sea correcta para las especies de amplio alcance, tales como felinos salvajes, es apropiado para el tamaño del ámbito hogareño promedio de la mayoría de las especies neo-tropicales consideradas aquí (Reid 2009). Sin embargo, la diversidad en la estructura de los sitios de las cámaras sirve para fortalecer la hipótesis de independencia, incluso para los felinos salvajes. Esto se debe a que las cámaras que no se instalan sistemáticamente cerca de elementos del hábitat que puedan atraer a la misma especie o grupo de especies de un sitio a otro (por ejemplo, algunas se instalan en los senderos, otras no; algunas se instalan cerca de almendros (Dipteryx panamensis), otras no. Además, varios estudios advierten que si el espacio de las cámaras es demasiado amplio, incluso para los felinos salvajes, ya que tiende a disminuir la probabilidad de detección y resultar en cálculos de densidad y abundancia bajos (Dillon \& Kelly, 2007). 
Figura No. 1: Distribución de una cámara, de los tres juegos en sitios en comparación con Laguna de Perlas. No se incluyen las cámaras de Karawala, se ubican más al norte, en este mapa.

\section{Análisis}

Las fotografías fueron inicialmente organizadas y resumidas mediante las cámaras automatizada de datos de software para el análisis automatizada de datos de trampas fotográficas que se detalla en Harris et al. (2010). El software requiere que cada foto tenga etiqueta que describa el nombre del sitio, las especies y la cantidad de individuos que aparece en la foto, y la fecha y hora en

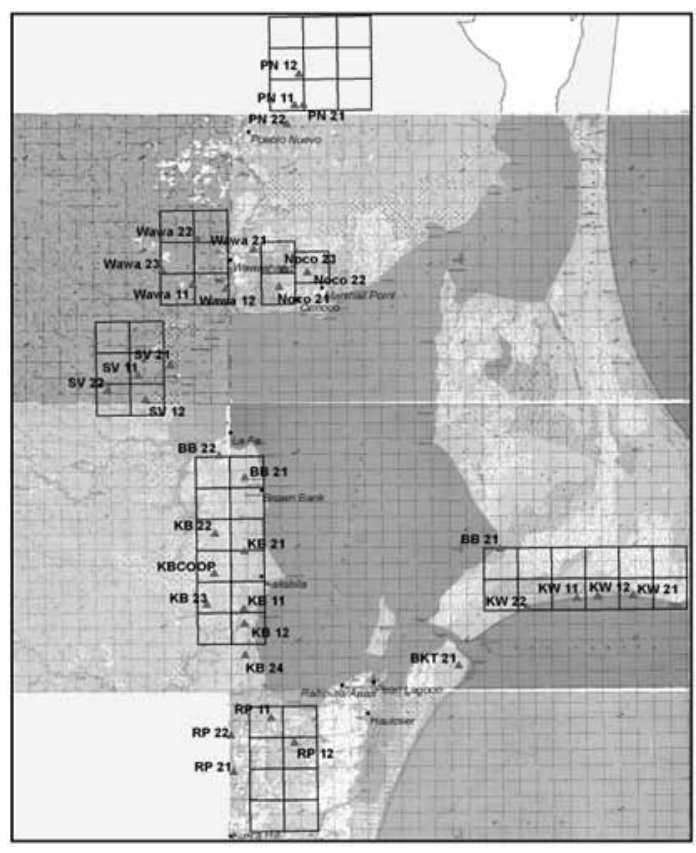
que se sacó. Entonces, el programa organiza las fotos de acuerdo a esta información, y las resume y analiza con una variedad de cálculos comunes y estimadores de interés (Harris et al., 2010). Algunos resultados reportados relacionados con la composición de comunidades de especies y con las variables tasas de detección vienen directamente de la producción del software. Otros fueron filtrados según su clasificación en la lista roja de IUCN (2010), o según varias medidas de distancia, pero de otra manera vienen directamente desde la misma salida.

Otras medidas de uso común para evaluar la diversidad y composición de la fauna de un sitio, son las curvas de acumulación de especies, se realizaron con el software EstimateS (Colwell, 2009). Para hacer estos cálculos es necesario muestrear varias veces en el mismo lugar. Esto se debe a la necesidad de hacer matrices en que el número de muestra $(1 \ldots \mathrm{n})$ va sobre las especies detectadas. Por ejemplo, el matrix

\begin{tabular}{cc}
\hline 1 & 0 \\
\hline 0 & 1 \\
\hline
\end{tabular}

Esto podría indicar que una zarigüeya (fila 1) se detectó en la muestra 1 (columna 1), pero no en la muestra 2 (columna 2), mientras que un mapache (fila 2) se detectó en la muestra 2, pero no en la muestra 1. Para calcular las curvas de cada de las comunidades (de personas), los datos de todas las cámaras de una comunidad se agruparon en una gran matriz de tal manera que las listas de especies (filas) representaba la diversidad total del sitio y cada día de cada cámara tenía su columna única. Para llenar la matriz utilizada en las curvas de las especies, el número de individuos que aparecen en las fotografías se utilizó en lugar de sólo los datos de incidencia. Esto se debe a que los 
estimadores de riqueza de especies que dan cuenta de la abundancia (en este caso el Chao-Jaccard y Chao-Sorrensen, estimadores basados en abundancia) son más adecuados para conjuntos de datos con numerosas especies raras, que son los estimadores basados en la incidencia tradicional. Muchas de nuestras especies se detectaron sólo en 1-3 puntos. Se ha demostrado que los estimadores más tradicionales son inexactos en estos casos y puede conducir a conclusiones incorrectas (Chao \& Colwell, 2005).

La biomasa promedia de la fauna de mamíferos detectados en las fotos por 100 días también se calculó para cada sitio de cámara $(1 \ldots$ j):

$$
\mathrm{A}_{\mathrm{j}}=\sum \mathrm{dr}_{\mathrm{i}, \mathrm{j}}{ }^{*} \mathrm{~m}_{\mathrm{i}}
$$

Con $\mathrm{dr}_{\mathrm{i}}$ definido como la tasa de detección por 100 días para la especie $i$ en el lugar j y m definido como la masa promedia de la especie $i$ de acuerdo con Reid (2009). Dos especies de aves, el pavón y el tinamú, también se incluyeron en este cálculo porque se reporta con frecuencia que estas aves grandes son vulnerables a la caza y la alteración del hábitat significativo (Smith, 2010). Una medida de la biomasa promedio fue elegido para la comparación entre sitios, porque en múltiples estudios se ha demostrado que disminuye con la destrucción del hábitat y la caza excesiva (al Brashares et al. 2004; Peres, 2000). En efecto, en igualdad de condiciones (ecológicas, geográficas, etc.) otras, si el conjunto de mamíferos de un lugar tiene una biomasa más baja en comparación con otro lugar, por lo general indica que el primer lugar tiene una mayor tasa de caza de ungulados grandes y / o tiene más destrucción del hábitat, y/o tiene más gente que mata a los grandes depredadores como es el jaguar. En este sentido, se puede utilizar esta medida para explicar mejor la disimilitud sitio, mejor que un índice simple (como el número de especies, por ejemplo). Inter-comunitaria comparación de las medias de la biomasa se realizó mediante procedimientos estadísticos no paramétricos, debido al tamaño pequeño de la muestra a nivel de cada comunidad.

La biomasa promedio de la fauna de mamíferos detectados en las fotos por 100 días, fue también utilizado como la variable dependiente en modelos de regresión que comprobó los hipótesis impulsando el segundo objetivo de esta investigación de una manera más formal. Estos modelos exploraron los impactos que la proximidad a la carretera de Laguna de Perlas y los efectos de la proximidad a la comunidad más cercana (ajustada por tamaño de la población de la comunidad en consideración) tienen sobre la vida silvestre detectada en cada sitio de la cámara. Aunque se trata de medidas básicas, se consideraron adecuadamente sensibles para informar futuras investigaciones. R, versión 2.11.1 (R. Development Core Team, 2010) fue utilizado para todos los procedimientos estadísticos que se realizaron fuera de estimaciones y el software de Harris (2010). 


\section{Resultados y discusión}

Tres cámaras fueron robadas y dos irreparablemente dañadas por el agua durante el primer año de la investigación. La mayoría funcionaron desde su instalación hasta su recuperación, aunque hubo varios que dejaron de tomar fotografías antes de la recuperación debido a la batería baja. El esfuerzo total fue de 4.935 días de trampa que produjo 1.792 eventos fotográficos independientes de 50 especies (ver Apéndice No. 1), además de numerosos fotos con perros, humanos, familias, o fotos sin animales que no se incluyeron o se analizaron. A medida que las especies más comunes con frecuencia pasan por las trampas de cámara varias veces en el mismo día, sólo las detecciones de una especie con intervalos mayores de 5 horas entre sí, se definieron como eventos independientes. La cantidad de tiempo elegido para abordar esta cuestión varía en la literatura por varias razones; sin embargo, cinco horas excede el intervalo elegido para otros inventarios de la fauna terrestre y, se consideró lo suficientemente largo como para asegurar la validez del supuesto de independencia.

Las curvas de acumulación de especies se calcularon para todas las comunidades y los cálculos de la riqueza de especies varían entre 14-23. Ejemplos de cuatro curvas se muestran a continuación para cuatro comunidades a diferentes distancias de la carretera de Laguna de Perlas (Figura No. 2). Rocky Point se refiere a la zona adyacente a la Laguna de Perlas, donde muchos comunitarios tienen sus fincas. Estas comunidades fueron elegidas para la comparación porque todos se muestrearon con una frecuencia más alta del promedio, lo que aumenta la precisión de las estimaciones, y cada uno se encuentra a una distancia distinta de la nueva carretera.

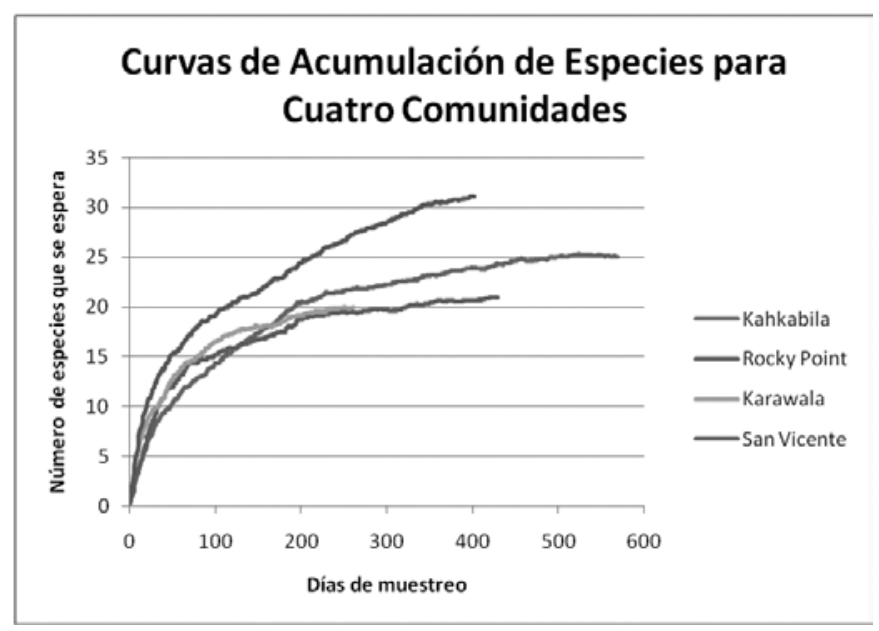

Figura No. 2: Curva de acumulación de especies para cuatro comunidades que se muestrearon de una manera parecida. Estas comunidades se ubican a distintas distancias de la carretera de Laguna de Perlas. En orden de distancia desde la nueva carretera: Rocky Point, Kahkabila, San Vicente y Karawala. 
La tasa de detección por 100 días en cada sitio de cámara para especies amenazadas, en peligro de extinción, y especies vulnerables $(\mathrm{n}=5)$ según la Lista Roja de la IUCN (2010) también se analizó en relación con la distancia de Laguna de Perlas (Figura No.3). El puma también se agregó a la lista como una especie de amplio alcance que requiere grandes extensiones de hábitat.

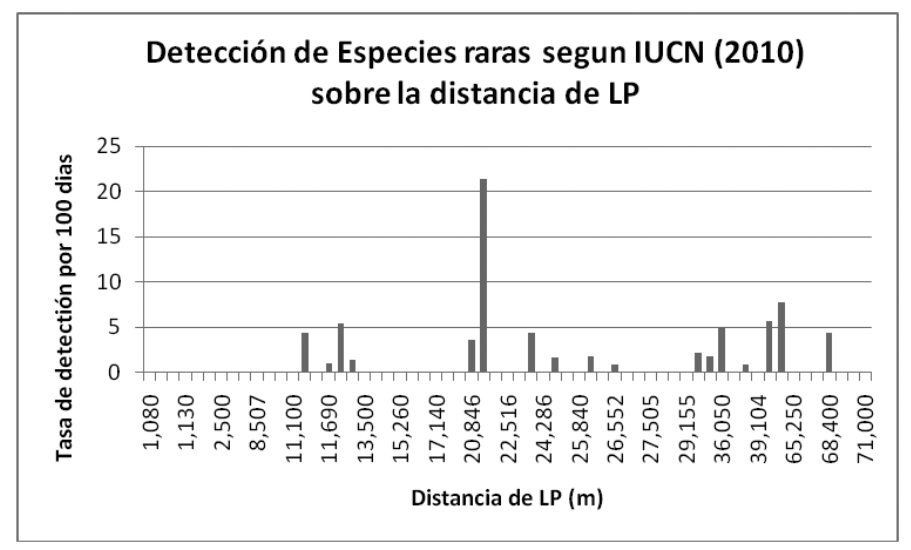

Figura No. 3: Tasa de detección por 100 días en relación con la distancia de la nueva carretera para especies casi amenazadas, especies vulnerables y especies en peligro de extinción según la Lista Roja de la UICN (2010). Los puntos de datos no son suficientes para un análisis estadístico.

El volumen de la biomasa promedio por 100 días, varió desde o hasta $764 \mathrm{~kg}$ por 100 días de trampa. Los resultados fueron utilizados en: 1) Una comparación de la biomasa de los sitios alrededor de las cuatro comunidades de la Figura No. 2 (Figura No. 5), 2) Una evaluación de la relación entre el promedio de biomasa por 100 días para de cada sitio de cámara y la distancia del sitio de la carretera de Laguna de Perlas (Figura No. 6), у 3) Un diagrama de puntos de la biomasa promedio por 100 días para todos los puntos de cámara sobre la distancia del sitio de la comunidad más cercana ajustado por tamaño de la población de esa comunidad (Figura No.7). Resultados pertinentes de las pruebas estadísticas se encuentran en el pie de las figuras, sin embargo el único modelo lineal estadísticamente significativa (en alfa $=0.01$ ) fue un modelo de segundo orden polinomio usado para describir el diagrama en Figura No. 7. Este modelo indica que, en igualdad de condiciones, valores bajos en las inmediaciones de una comunidad, valores altos en las distancias intermedias, y los valores más bajos en los lugares más distantes se puede esperar. 

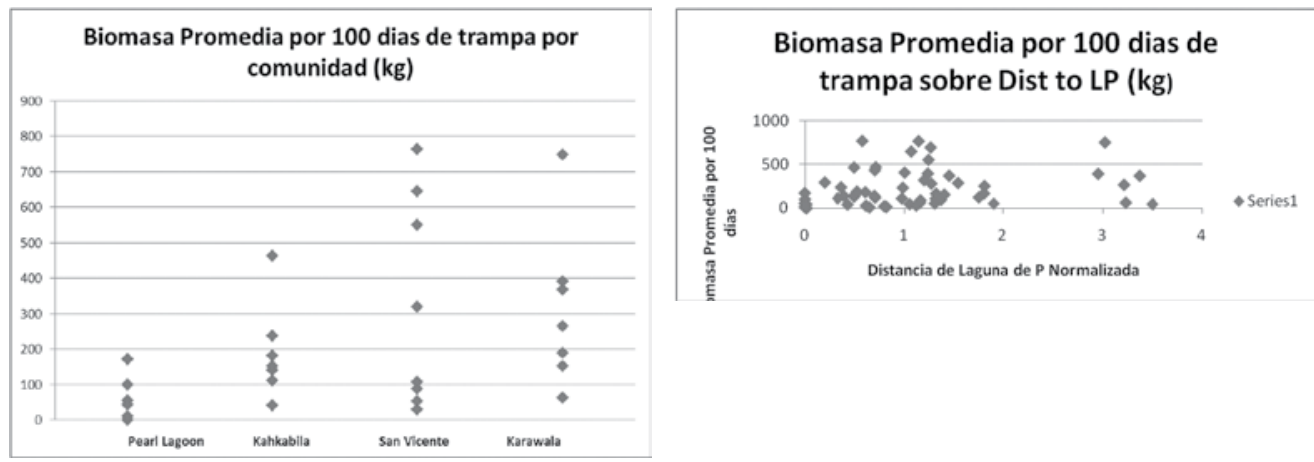

Figura No. 4: La biomasa promedio por 100 dias de trampa de las mismas cuatro comunidades que se describen en la figura 3. Promedios para cada comunidad son Laguna de Perlas: 63.51 * Kahkabila t: 180.74, San Vicente: 319.89, y Karawala: 277,69. ( ${ }^{*}=$ Significativamente diferente de todos los demás promedios en alfa $=0.05, p=0,02($ Kahk), $p=0,03(S V)$ y $\mathrm{p}=0,02$ (Kara); = significativamente diferente de Karawala † promedia en alfa $=0.05$, $\mathrm{p}=0,4$; prueba $U$ de Mann-Whitney).
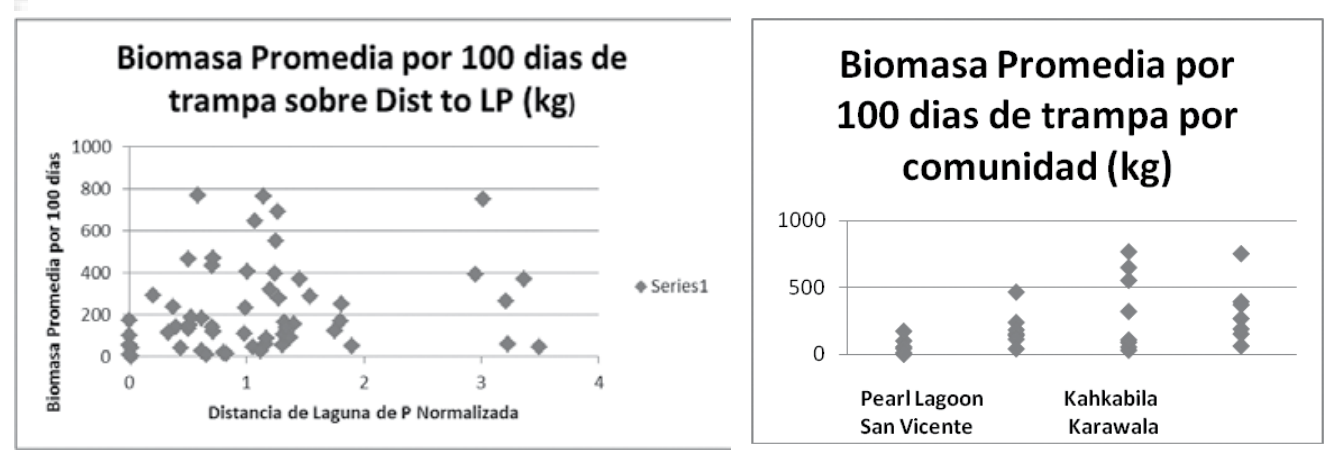

Figura No. 5: El mismo gráfico, sino como un diagrama de puntos y con la biomasa de todos los sitios de cámara sobre su distancia de la carretera de Laguna de Perlas; las comunidades no están etiquetados. Las dos medidas no son significativamente correlacionados, ni un modelo polinomial de segundo orden de ajustar los datos. Este diagrama incluye todos los sitios de cámara, incluyendo los ecosistemas de sabana y los sitios no comunitarios. Quitar estos sitios no comunitarios y ajustar los demás por tamaño de la población de comunidad no hace que sea significativa la correlación entre biomasa y distancia de Laguna de Perlas. 

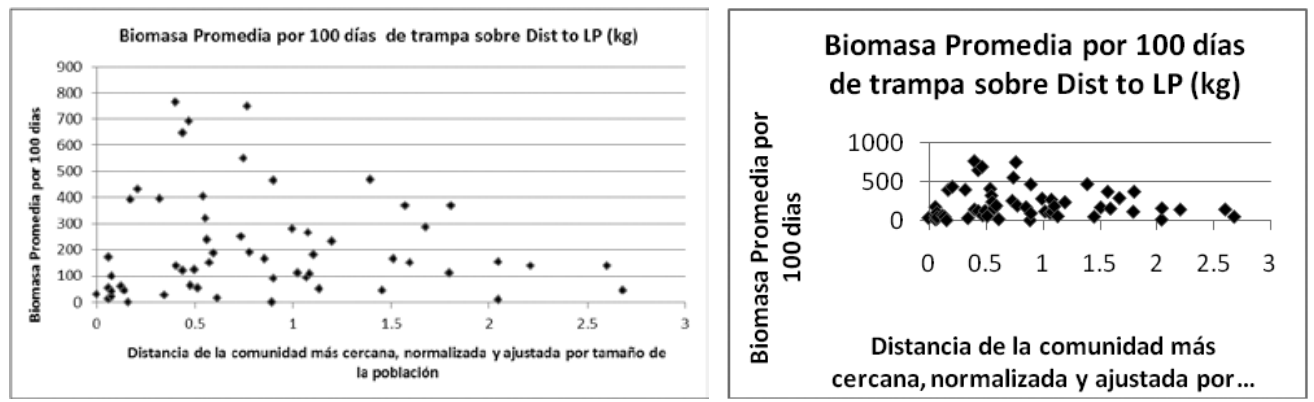

Figura No. 6: La media de biomasa sobre la distancia de la comunidad más cercana para todos los puntos de cámara en bosques comunitarios. Algunos sitios de manglar y bosque de ribera del pantano fueron excluidos como varios comunitarios reportan que no se utilizan por las comunidades y son relativamente inaccesibles. En este caso, una ecuación polinómica de segundo orden es significativo en alfa =0,01, aunque R2 = 0,15 , lo que indica, como se esperaba, que hay bastante variación en la medida de biomasa que no se explica por esta simple medida de distancia ajustada por tamaño de la población.

Objetivo 1: Al igual que en muchos lugares con niveles significativos de hábitat secundario, los bosques que rodean las comunidades del estudio aparecen albergar una alta densidad de especies ampliamente consideradas comunes, tales como pacas de tierras bajas, zarigüeyas comunes, guatusas de América Central, tlacuache grises de cuatro ojos, monos capuchinos blancos, y coatíes de hocico blanco. Sin embargo, cincuenta especies identificables apareció en las fotos, incluyendo varias que se consideran sensibles a la degradación del hábitat y / o caza excesiva: cuatro especies de felinos salvajes, aves raras, varios individuos de tapires, y manadas de pecaríes de labios blancos. Los datos preliminares parecen indicar la presencia de una fauna relativamente intacta. Esta conclusión está respaldada además por el diseño de nuestro estudio que sitúa cámaras dentro y fuera de pista. De hecho, varias especies raras e importantes en el mundo de la conservación, incluyendo jaguares, tapires, y ocelotes utilizan preferentemente los senderos para la dispersión, lo que significa que nuestros datos de la cámara puede estar subestimando la prevalencia de estas especies raras en el paisaje. Además, mientras que la curva de acumulación de especies alcanzó la asíntota, existen numerosas especies arbóreas que no se detectaron debido a nuestra metodología de muestreo, por lo que el conjunto completo de la fauna terrestre se compone de un número aún mayor de especies.

Aunque ninguna de las especies raras se detectó en los bosques adyacentes a Laguna de Perlas, todos fueron detectados adentro de los 20 kilómetros, sin duda dentro de los rangos de dispersión de los grandes mamíferos. Además, según lo indicado por las curvas de acumulación de especies y el volumen de la biomasa promedio por cada 100 días, ningún bosque comunitario carece de vida silvestre, lo que sugiere, para los 
felinos en particular, que la presa es lo suficientemente abundante para que la región le sirva en su función como corredor biológico.

Objetivo 2: Hay varias líneas de evidencia que sugieren que la nueva carretera de Laguna de Perlas y el desarrollo relacionado afectan negativamente a la fauna terrestre. En primer lugar, la Figura No. 4, en particular, revela cómo la biomasa promedio es significativamente menor en Rocky Point que en otros bosques comunitarios, comparables en términos de cantidad de sitios de cámara y cobertura espacial alcanzados por las cámaras. La diferencia más evidente entre estos bosques es la distancia de la carretera. Como se describió anteriormente, si un sitio tiene una menor biomasa promedio de otros sitios que son ecológicamente y geográficamente similares, por regla general, connota una mayor tasa de destrucción del hábitat, o la caza excesiva ( Brashares et al. 2004; Peres, 2000). Estas Conclusiones se apoyan más directamente por la observación de que ninguna de las especies que figuran como casi amenazadas, en peligro o deficiencia de datos se detectó a $11 \mathrm{~km}$ de la carretera nueva.

La segunda línea de evidencia es que los resultados preliminares sugieren que la fauna es más intacta mientras un sitio es más lejos de Laguna de Perlas. Por ejemplo, las fotos de los rebaños pecarí de labio blanco, cerca de Kahkabila nunca muestran más de 7 miembros de la manada, mientras muestran mucho más cerca de comunidades más lejanas.

La evidencia presentada hasta ahora, como se esperaba, no permite una conclusión definitiva acerca de la causalidad de estos patrones en la detección de la vida silvestre, los patrones observados coinciden con los patrones descritos en estudios anteriores sobre la relación entre el desarrollo de carreteras y la subsiguiente degradación permanente del medio ambiente (Laurence et al. 2009). De hecho, en muchos casos a nivel mundial, las carreteras, en general, se ha proporcionado un ingreso más fácil a los bosques y recursos forestales, lo que permite una taza de caza mayor y más deforestación, y que causa patrones como los observados en la RAAS (Laurence et al. 2009). Además, evidencia anecdótica sobre la degradación ambiental causada por el desarrollo recopilado en el proceso de instalar cámaras relacionado con la carretera es difícil de ignorar. Por ejemplo, en la región de Rocky Point, que es atravesada por la carretera, se dice que la deforestación para hacer finca es mucho más extensiva que en el norte de la carretera. En una entrevistas de modelos mentales que se llevaron a cabo por CAJ, antiguos cazadores de la zona de Rocky Point con frecuencia reportan que el esfuerzo requerido para la captura de las especies preferidas de alimentos tales como el venado de cola blanca y guiya de tierras bajas es mucho mayor que el esfuerzo requerido en tiempos anteriores a la carretera.

También hay evidencia basada en los análisis de las fotos que trata el segundo componente del objetivo 2, el análisis de los efectos de la comunidad más cercana a las poblaciones silvestres. De hecho, incluso en torno a comunidades distantes de la 
nueva carretera, la fauna terrestre se ve afectado por los procesos de desarrollo. La causa principal y más fuerte parece ser la frontera agrícola. Por ejemplo, la Figura No. 6 muestra que el aumento de distancia de las comunidades costeras ajustado por tamaño de la población no indica la lejanía cada vez mayor de la degradación de hábitat y por eso un aumento en la actividad de la fauna. Por el contrario, el aumento en distancia de una comunidad costera, después de un valor intermedio, connota una disminución sustancial en la biomasa promedio por 100 días de trampa. Como todos los puntos de la cámara se utilizaron en este análisis, parece que este fenómeno es una tendencia general en toda el área de estudio. Aunque este patrón se podría vincular a la variación geográfica o ecológica, esto es poco probable. La frontera agrícola es una hipótesis mucho más convincente ya que no hay evidencia de una barrera constante que corre por toda la costa que causaría el patrón patrones. Sin embargo, se descubrió bastante evidencia de la caza de mestizos durante la instalación de las trampas fotográficas en las cercanías de casi todas las comunidades. Ejemplos incluyen: la venta de carne de tapir en Karawala, encontrando los restos de un pavón cerca de una granja mestiza detrás Kahkabila, y evidencian las anécdotas que hay mestizos que han estado matando jaguares, tanto en la Laguna de Perlas como en Kahkabila. Como ocurre por las fronteras agrícolas en otros lados del mundo, los mestizos en la RAAS con la práctica de la queman y tumba para la cría de ganado, a gran velocidad y de manera permanente, destruyen los bosques y pantanos y por eso el hábitat de los animales terrestres. De este modo, se espera que su omnipresencia en el paisaje resulte en los patrones observados.

Es importante destacar, que un modelo lineal polinomio de segundo orden fue significativo para este conjunto de datos en alfa $=0.01$, lo que sugiere que tanto las comunidades costeras y las comunidades mestizas tienen un efecto negativo sobre la fauna local. Esta propuesta fue apoyada también por la observación de que casi todas las especies raras y sensibles a la modificación de su hábitat salieron en los sitios de distancias intermedias de las comunidades. Que esta distancia es de aproximadamente $5 \mathrm{~km}$ en promedio no es un buen indicador para la función de corredor de la región, como la literatura describe que el territorio típico de una comunidad de cazadores es igual a un círculo área con un radio de $10 \mathrm{~km}$ en torno al pueblo de origen (Alvard et al., 1997). Si esta generalización se aplica en la RAAS, esto implicaría que el territorio de los cazadores mestizos y los cazadores de las comunidades costeras se sobreponen, lo que disminuiría drásticamente la existencia de hábitat núcleo y por eso la densidad de especies que requiere de este tipo de habitat.

Sin embargo, estos primeros estudios no deben interpretarse como concluyentes. Tal vez la razón más evidente de esto es el alto grado de variación en todos los análisis. Por ejemplo, mientras que una comparación de la biomasa promedio por 100 días de trampa de algunos bosques comunitarios que son ecológicamente similares y difieren sobre todo en la distancia de la nueva carretera, que mostró una diferencia significativa, la relación no fue estadísticamente significativa cuando la comparación 
incorporó todos los sitios de cámara. Incluso el análisis de la biomasa promedio por 100 días y la distancia de la comunidad más cercana ajustada por tamaño de la población local no es concluyente. Aunque el modelo lineal polinomio de segundo orden es significativo, es muy claro que el diagrama de puntos tiene mucha variación que no se puede explicar con una medida de distancia. Es por eso que el modelo tiene un $\mathrm{R}^{2}=$ 0,15 . Esto no hace nada para refutar el argumento de que los cambios causados por la construcción de carreteras está causando una tensión en la vida silvestre. De hecho, que un proxy tan sencillo era capaz de dar resultados significativos indirectamente puede apoyarlo. Sin embargo, lo que sin duda hace es describir la construcción de carreteras, el desarrollo posterior, y los cambios ecológicos como procesos demasiado complejos para tratar con un proxy simple e indicadores unidimensionales. Esto implica obviamente que la determinación de las causas de los cambios ambientales en el contexto del desarrollo de la RAAS en los próximos años requerirá de múltiples medidas interdisciplinarias para un estudio más amplio. Esto es especialmente cierto dada la complejidad cultural, ecológica y política de la RAAS.

Una segunda razón por la cual es difícil aceptar estas conclusiones como algo definitivo (y, de hecho, poco inteligente) suponer que un patrón indica un proceso en particular (MacKenzie et al. 2005). Hay pocos datos sobre la fauna terrestre de 10 años, 15 años, o incluso 2 años atrás. Esto significa que actualmente no hay manera de saber con certeza que los datos de las cámaras son diferentes de los datos del 2006, antes de la construcción de carreteras y por lo tanto no hay manera de saber con certeza cómo esta infraestructura y el desarrollo causado por ella va afectando la vida silvestre en la RAAS. El único medio para llegar a Conclusiones definitivas sobre la relación entre la carretera Laguna de Perlas, el desarrollo y los ecosistemas terrestres, será tomar medidas repetidas en los próximos años. A pesar de estas incertidumbres, otra declaración definitiva proporcionada por los datos es que, sea debido a la construcción de la nueva carretera o no, el refugio para la fauna tan a menudo descrito en la literatura como en impecable estado ya no es tan remota, por lo menos en las inmediaciones de la Laguna de Perlas. Como un vínculo potencialmente clave en el Corredor Biológico Mesoamericano, esto es importante saber para los conservacionistas.

\section{Conclusión}

Este informe proporciona datos que sirven como una línea base y que resultarán fundamentales en el seguimiento ecológico a largo plazo en la RAAS y previsto para los próximos 5 años. Los resultados también ofrecen sugerencias para los administradores de recursos naturales e investigadores que trabajan las regiones de desarrollo rápido. La construcción de una carretera que conecta un lugar con nuevas influencias y mercados y la degradación del medioambiente es bastante complejo y depende de múltiples variables que actúan en múltiples escalas. En particular, es claro que hay impactos regionales del desarrollo que afectan a la fauna silvestre, pero también existe una gran cantidad de variación de la escala más local que amortigua o intensifica 
estas influencias para determinar el efecto final. Esto sugiere que cualquier medida de conservación, la gestión ambiental, o una iniciativa de investigación que pretende abordar cuestiones del desarrollo rural y los cambios ambientales relacionados con ello tienen que ocurrir en varias escalas.

\section{Lista de referencias}

Alvard, M.S., J.G. Robinson, K.H. Redford, and H. Kaplan (1997). The sustainability of subsistence hunting in the neotropics. Conservation Biology. 11(4):977-982.

Archbold, M. (2010). Conservation of Green Turtles on the Caribbean Coast of Nicaragua. Lap Lambert Academic Publishing, 2010. pp. 128

Barret, B. (1994). Medicinal plants of Nicarqaguas Atlantic Coast. Economic botany. 48(1):8-20.

Bradford, D., M. Castrillo, R. Guevara, D. Gutierrez, Z. Ramos, \& J. Sing. 2003. Estado actual de la fauna de la Cuenca del Rio Escofram doce años después del huracán Juana. WANI: Revista del Caribe Nicaragüense. 32:49-60

Brashares, J.S., P. Arcese, M.K. Sam, P.B. Coppolillo, A.R.E. Sinclair \& A. Balmford (2004). Bushmeat hunting, wildlife declines and fish supply in West Africa. Science 306, 1180-1183.

Campbell, C.L. (2003). Population assessment and management needs of a green turtle, Chelonia mydas, population in the western Caribbean. $\mathrm{PhD}$ thesis, University of Florida, Gainesville, USA.

Carr, A. (1956). The windward road. $1^{\text {st }}$ edition. Gainesville: University of Florida Press.

Chao, A., Chazdon, R. L., Colwell, R. K., \& Shen, T.-J. (2005). A new statistical approach for assessing similarity of species composition with incidence and abundance data. Ecology Letters 8, 148-159.

Christie, P., D. Bradford, R. Garth, B. Gonzalez, M. Hostetler, O. Morales, R. Rigby, B. Simmons, E. Tinkham, G. Vega, R. Vernooy, N. White. (2000). TAKING CARE OF WHAT WE HAVE Participatory Natural Resource Management on the Caribbean Coast of Nicaragua. IDRC/CIDCA. Pp. 188

Coe, F.G. (2008). Ethnobotany of the Rama of Southeastern Nicaragua and Comparisons with Miskitu Plant Lore. Economic Botany 62(1): 40-59 
Cody, M. Antbird guilds in the lowland Caribbean rainforest of Southeast Nicaragua. The Condor. 102:784-794.

Colwell, R. K. (2009). EstimateS, Version 8.2: Statistical Estimation of Species Richness and Shared Species from Samples (Software and User's Guide).

Dillon, A. \& M.J. Kelly. Ocelot Leopardus pardalis in Belize: the impact of trap spacing and distance moved on density estimates. Oryx. 41(4):469-477.

Garland, K.A. \& R.R. Carthy. Changing taste preferences, market demands and traditions in Pearl Lagoon, Nicaragua: a community reliant on green turtles for income and nutrition. Conservation and Society. 8(1):55-72

Harmsen, B.J., Foster, R.J., Silver, S.C., Ostro, L.E.T. \& Doncaster, C.P. 2010. Differential Use of Trails by Forest Mammals and the Implications for Camera Trap Studies, a Case Study From Belize. Biotropica, 42: 126-133

Harris, G, R. Thompson, J.L. Childs, and J.G. Sanderson. 2010. Automatic Storage and Analysis of Camera Trap Data. Bulletin of the Ecological Society of America 91:352-360.

IUCN Red List. (2010). Official Home Page: http://www.iucnredlist.org/ [Accessed 19 November 2010]

Jamieson, M. (1999). Poverty among the indigenous peoples of Nicaragua. Indigenous Peoples and Community Development Unit. Inter-American Development Bank. Sustainable Development Department. Pp. 71

Jamieson, Mark. (2002). Ownership of sea shrimp production and perceptions of economic opportunity in a Nicaraguan Miskitu village. Ethnology 41(3): 281-298.

Jamieson, M. (2010). Bloodman, Manatee Owner, and the destruction of the Turtle Book: Ulwa and Miskitu representations of knowledge and the moral economy. Journal of the Royal Anthropological Institute. 16(1):31-45.

Jimenez, I. (2002). Heavy poaching in prime habitat: the conservation status of the West Indian manatee in Nicaragua. Oryx 36:272-278

Laurance, W.F., M. Goosem, and S.G.W. Laurance. Impacts of road and linear clearings on tropical forests. Trends in Ecology \& Evolution. 24(12):659-669

Loveland, F. O. (1976). Tapirs and manatees: cosmological categories and social process among Rama Indians of eastern Nicaragua. Pp. 67-82 in Frontier Adaptations in 
Lower Central America, M. W. Helms and F. O. Loveland (eds.). Philadelphia Institute for the Study of Human Issues. Philadelphia, PA. 178 pp.

MacKenzie, D.I., J.D. Nichols, J.A. Royle, K.H. Pollock, J.E. Hines and L.L. Bailey. (2005). Occupancy estimation and modeling: inferring patterns and dynamics of species occurrence. Elsevier, San Diego, USA.

Nietschmann, B. (1973). Between land and water: the subsistence ecology of the Miskito Indians, eastern Nicaragua. $1^{\text {st }}$ Edition. New York Seminar Press.

Peres, CA. (2001). Synergistic effects of subsistence hunting and habitat fragmentation on Amazonian forest vertebrates. Conservation Biology. 15(6):1490-1505.

R Development Core Team (2005). R: A language and environment for statistical computing, reference index version 2.x.x. R Foundation for Statistical Computing, Vienna, Austria. ISBN 3-900051-07-0, URL http://www.R-project.org.

Reid, F.A. (2009). A field guide to the mammals of Central America and Southeast Mexico. Oxford University Press, USA; 2 edition. Pp. 384.

Schmitt, K.M. and D.B. Kramer. Road development and market access on Nicaragua's Atlantic coast: implications for household fishing and farming practices. Environmental Conservation. 36:289-300

Smith, D.A. (2010). The harvest of rainforest birds by indigenous communities in Panama. Geographical Review. 100(2):187-203.

Sollis, P. (1989). The Atlantic Coast of Nicaragua: development and autonomy. Journañ of Latin American Studies. 21(3):481-520

Vandermeer, J., and I. Perfecto (2005). A breakfast of biodiversity: The true causes of rain forest destruction (second edition). (Vandermeer and Perfecto). Institute for Food and Development Policy. 


\section{Apéndice No. 1}

Tabla No. 2: Especies claves de aves y mamíferos que salieron en las fotos.

\begin{tabular}{ll}
\hline Mamíferos & Aves \\
\hline - Cuniculus paca & \\
- Dasyprocta punctata & \\
- Dasypus novemcinctus & \\
- Didelphis marsupialis & \\
- Procyon lotor & - Columbina talpacoti \\
- Nasua narica & - Tinamus major \\
- Conepatus leuconotus & - Tigrisoma mexicanum \\
- Odocoileus virginianus & - Mesembrinibis cayennensis \\
- Pecari tajacu & - Momotus momota \\
- Tayassu pecari & - Leucopternis albicollis \\
- Philander opossum & - Ortalis vetulaMoc orop \\
- Cebus albifrons & - Amaurolimnas concolor \\
- Leopardos pardalis & - Crax rubra \\
- Mazama americana & - Aramides cajanea \\
- Eira barbara & - Piaya cayana \\
- Tamandua mexicana & - Buteogallus urubitinga \\
- Pantera onca & - Mycteria americana \\
- Puma yagouaroundi & \\
- Tapirus bairdii & \\
\hline
\end{tabular}

